\title{
Synthesis of 3-(o-stilbenyl)sydnone and 3-(o-stilbenyl)-4-substituted- sydnone derivatives and their antitumor evaluation
}

\author{
Kristina Butković, ${ }^{\text {a,c }}$ Željko Marinić, ${ }^{b}$ and Marija Šindler-Kulyk ${ }^{a *}$ \\ ${ }^{a}$ Department of Organic Chemistry, Faculty of Chemical Engineering and Technology, \\ University of Zagreb, Marulićev trg 19, 10000 Zagreb, Croatia \\ ${ }^{b}$ Center for NMR, Rudjer Bošković Institute, Bijenička cesta 54, 10000 Zagreb, Croatia \\ ${ }^{c}$ Present address: Galapagos istraživački centar, Prilaz baruna Filipovića 29, \\ 10000 Zagreb, Croatia \\ E-mail: marija.sindler@fkit.hr
}

\begin{abstract}
A series of novel stilbene-sydnone derivatives were synthesized by the following sequence of reactions: starting from methyl anthranilate via glycine- and nitrosoglycine derivatives the corresponding 3-(o-carbomethoxyphenyl)-4-H/Me/Ph-sydnones were prepared and transformed to 3-(o-formylphenyl)-4-H/Me/Ph-sydnones, starting materials for Wittig reaction with various phosphonium salts to stilbenylsydnone derivatives. Final products were evaluated for their cytotoxic properties on five cancer cell lines, whereby the cis-4-methyl-3-[2-[2-(4methylphenyl)ethenyl]phenyl]sydnone $\mathbf{5}$ and cis-4-phenyl-3-[2-[2-(4-chlorophenyl)ethenyl]phenyl]sydnone $\mathbf{1 0}$ showed the most pronounced activity.
\end{abstract}

Keywords: Stilbenes, sydnones, synthesis, antitumor evaluation

\section{Introduction}

Sydnones ${ }^{1}$ are five-membered heterocycles. They belong to a class of dipolar compounds known as "mesoionic" and can be represented as hybrids of a number of mesomeric ionic forms (Figure 1). Since their first synthesis ${ }^{2}$ diverse substituted sydnones have been synthesized and many of them have shown useful biological properties ${ }^{3}$ (e.g., as antibacterial, ${ }^{4}$ antineoplastic ${ }^{5}$ and antiinflammatory ${ }^{6}$ agents). In an effort to prepare new sydnones of potential pharmacological activity, especially the combination of the sydnone moiety with some other pharmacophore, we turned our attention to the preparation of stilbene substituted sydnones. Stilbene-based compounds are widespread in nature and have become of particular interest to chemists and biologists because of their wide range of biological activities. ${ }^{7}$ Stilbene itself does not occur in 
nature, but hydroxylated stilbenes ${ }^{8,9}$ have been found in many medicinal plants. We expected that the structures obtained by combining the stilbene and sydnone moieties might possess valuable biological activity (Figure 2).
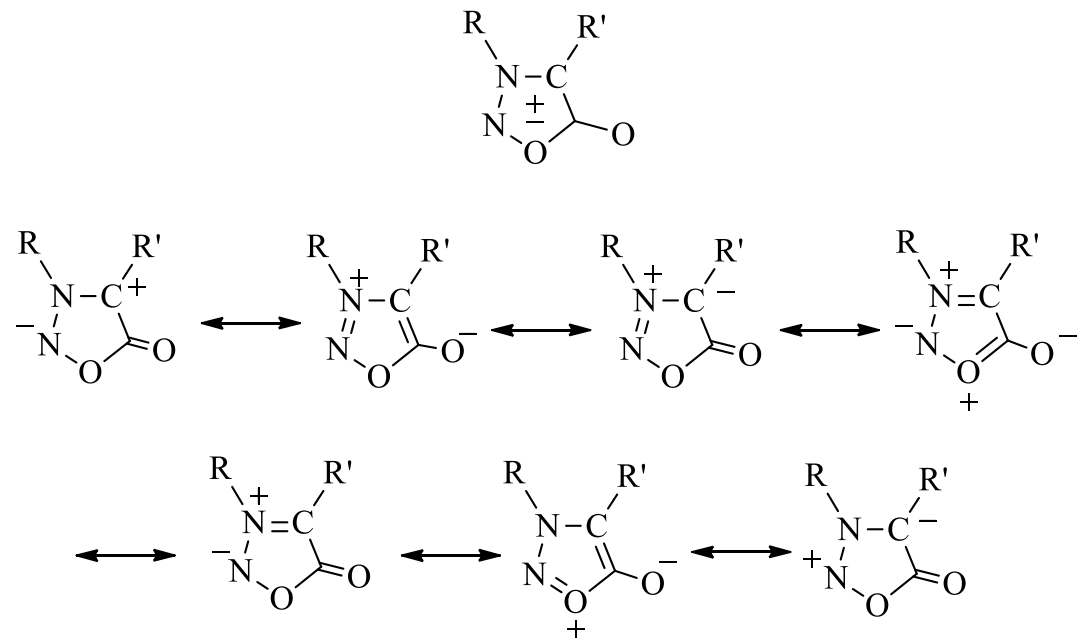

\section{Figure 1}

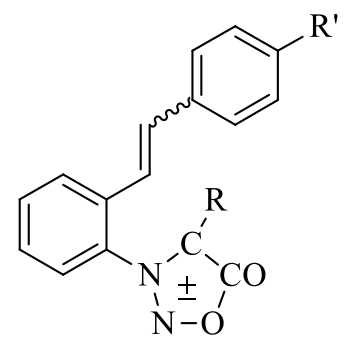

$$
\begin{aligned}
& 1 \mathrm{R}=\mathrm{H}, \mathrm{R}^{\prime}=\mathrm{CH}_{3} \\
& 2 \mathrm{R}=\mathrm{H}, \mathrm{R}^{\prime}=\mathrm{Cl} \\
& 3 \mathrm{R}=\mathrm{H}, \mathrm{R}^{\prime}=\mathrm{Br} \\
& 4 \mathrm{R}=\mathrm{H}, \mathrm{R}^{\prime}=\mathrm{OCH}_{3}
\end{aligned}
$$

$$
\begin{aligned}
& 5 \mathrm{R}=\mathrm{CH}_{3}, \mathrm{R}^{\prime}=\mathrm{CH}_{3} \\
& 6 \mathrm{R}=\mathrm{CH}_{3}, \mathrm{R}^{\prime}=\mathrm{Cl} \\
& 7 \mathrm{R}=\mathrm{CH}_{3}, \mathrm{R}^{\prime}=\mathrm{Br} \\
& \mathbf{8} \mathrm{R}=\mathrm{CH}_{3}, \mathrm{R}^{\prime}=\mathrm{OCH}_{3}
\end{aligned}
$$

$9 \mathrm{R}=\mathrm{Ph}, \mathrm{R}^{\prime}=\mathrm{CH}_{3}$

$10 \mathrm{R}=\mathrm{Ph}, \mathrm{R}^{\prime}=\mathrm{Cl}$

$11 \mathrm{R}=\mathrm{Ph}, \mathrm{R}^{\prime}=\mathrm{Br}$

$12 \mathrm{R}=\mathrm{Ph}, \mathrm{R}^{\prime}=\mathrm{OCH}_{3}$

Figure 2

In the present paper we describe the synthesis of $c i s$ - and trans-3-(o-stilbenyl)sydnones 1-4 and cis- and trans-3-(o-stilbenyl)-4-substituted-sydnones 5-12, a system in which are associated two pharmacophore groups, stilbene and sydnone, as potential new antitumor agents. 


\section{Results and Discussion}

\section{Chemistry}

The cis/trans-stilbene-sydnones 1-12 were chosen in order to investigate the configurational influence of the stilbene moiety as well as the substituent effects (structure-activity relationship SAR) on their biological behaviour. The first example of the stilbene moiety attached to sydnone ring was described by us previously and compounds 1, 5 and 9 have been prepared from the corresponding stilbene derivatives by closing the sydnone ring at the end of the synthetic pathway. ${ }^{10}$ Due to the needs in this work for a whole series of stilbene-sydnone derivatives in both cis- and trans-configurations, with different substituents in position 4' of the stilbene moiety and position 4 of the sydnone moiety, it was necessary to elaborate a more efficient route than the one described previously. In this work a different synthetic approach to sydnones 1-12 is elaborated according to Scheme 1.
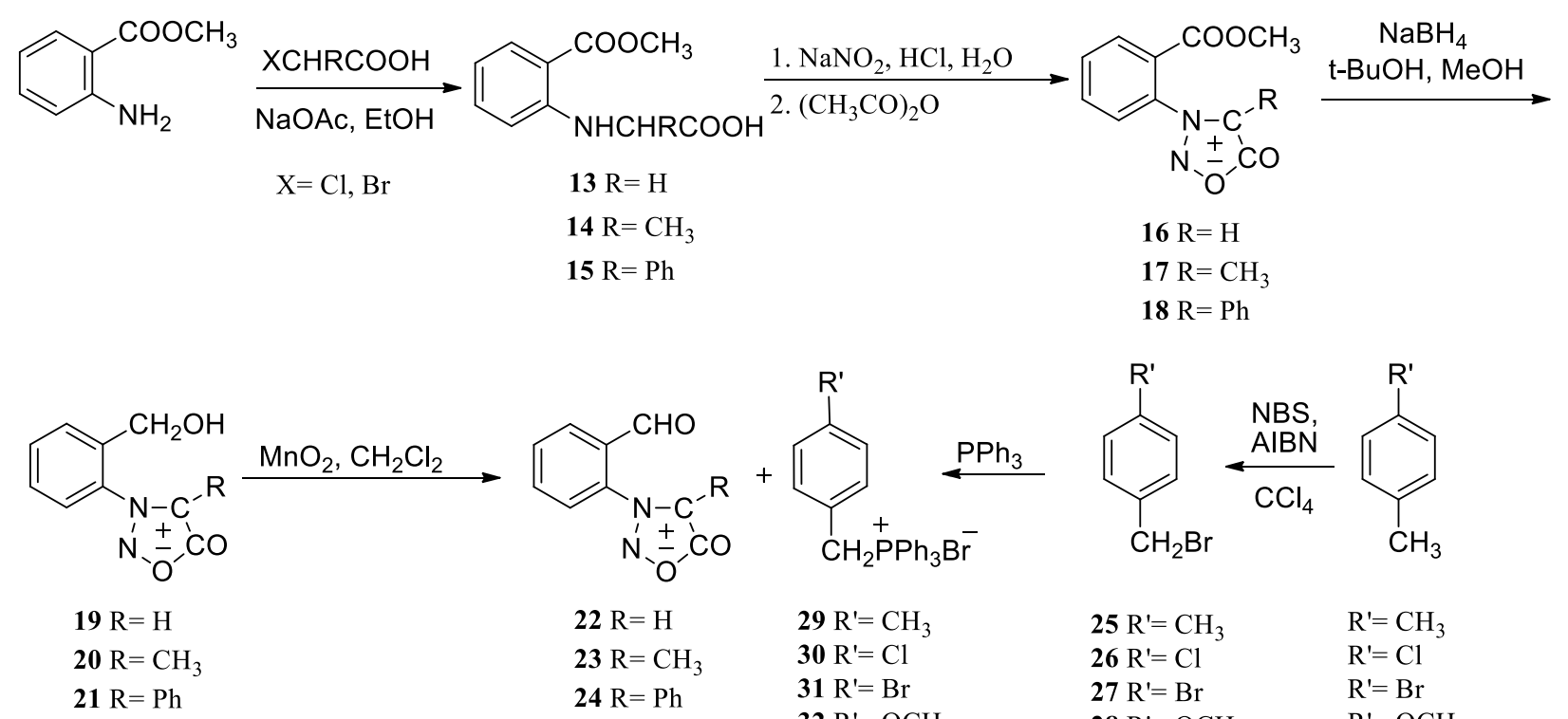
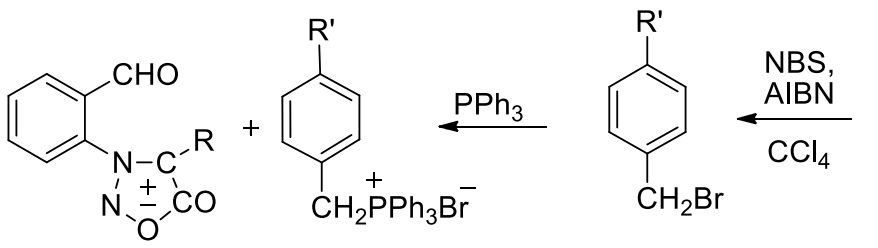<smiles>[R]c1ccc(C)cc1</smiles>

$22 \mathrm{R}=\mathrm{H}$ $23 \mathrm{R}=\mathrm{CH}_{3}$ $29 \mathrm{R}^{\prime}=\mathrm{CH}_{3}$ $30 \mathrm{R}^{\prime}=\mathrm{Cl}$ $24 \mathrm{R}=\mathrm{Ph}$

$25 \mathrm{R}^{\prime}=\mathrm{CH}_{3}$ $26 \mathrm{R}^{\prime}=\mathrm{Cl}$ $27 \mathrm{R}^{\prime}=\mathrm{Br}$ $32 \mathrm{R}^{\prime}=\mathrm{OCH}_{3}$<smiles>[R]O[N+]([O-])(O[Na])OCC</smiles>

$$
\begin{aligned}
& 1 \mathrm{R}=\mathrm{H}, \mathrm{R}^{\prime}=\mathrm{CH}_{3} \\
& 2 \mathrm{R}=\mathrm{H}, \mathrm{R}^{\prime}=\mathrm{Cl} \\
& 3 \mathrm{R}=\mathrm{H}, \mathrm{R}^{\prime}=\mathrm{Br} \\
& 4 \mathrm{R}=\mathrm{H}, \mathrm{R}^{\prime}=\mathrm{OCH}_{3}
\end{aligned}
$$

$$
\begin{aligned}
& 5 \mathrm{R}=\mathrm{CH}_{3}, \mathrm{R}^{\prime}=\mathrm{CH}_{3} \\
& \mathbf{6} \mathrm{R}=\mathrm{CH}_{3}, \mathrm{R}^{\prime}=\mathrm{Cl} \\
& 7 \mathrm{R}=\mathrm{CH}_{3}, \mathrm{R}^{\prime}=\mathrm{Br} \\
& \mathbf{8 R} \mathrm{R}=\mathrm{CH}_{3}, \mathrm{R}^{\prime}=\mathrm{OCH}_{3}
\end{aligned}
$$

Scheme 1 
The first step involves the reaction of methyl anthranilate with halogenoacetic acids to give $N$-(o-carbomethoxy)-aminoacids 13-15. The resultant aminoacids were treated with sodium nitrite and the crude $N$-nitroso derivatives submitted to dehydration with acetic acid anhydride to give $N$ - $\left(o\right.$-carbomethoxyphenyl)sydnones $16,{ }^{11} \mathbf{1 7}$ and 18. The sydnone derivatives were purified by column chromatography and isolated in $46 \% \mathbf{1 6}, 59 \% 17$ and $21 \% \mathbf{1 8}$ yields. After reduction with $\mathrm{NaBH}_{4}$ in methanol/t-butanol to hydroxymethyl derivatives 19-21, followed by oxidation with $\mathrm{MnO}_{2}$ and filtration through the column with silica gel, the formyl derivatives $\mathbf{2 2 - 2 4}$ were obtained in 61-68\% yield. The resulting 3-(2-formylphenyl)sydnone 22, 3-(2-formylphenyl)-4methylsydnone 23 and 3-(2-formylphenyl)-4-phenylsydnone $\mathbf{2 4}$ were the starting compounds for the continuation of the synthesis to stilbene-sydnone derivatives 1-12. The obtained formylphenylsydnone derivatives 22-24 were submitted to Wittig reaction with $p$-substitutedbenzyltriphenylphosphonium salts 29-32, which were prepared from the corresponding $p$ substituted-benzylbromides 25-28 and triphenylphosphine in toluene solution, by a standard procedure. The Wittig reactions were performed in ethanol with the addition of sodium ethoxide as a base. All sydnone derivatives were prepared as mixtures of trans- and cis-isomers, which were purified and separated by consecutive column chromatography.

All prepared compounds are identified and characterized by spectroscopic methods. Derivatives 14 and 15 have mass spectral molecular ions at $m / z, 224$ and 286, respectively, and in their IR spectra exhibit two bands at 1694-1702 and 1667-1685 $\mathrm{cm}^{-1}$, which correspond to acid and ester carbonyl groups. Also, their NMR spectra correspond to those expected for the proposed structures. The $N$-(o-carbomethoxyphenyl)sydnone derivatives $\mathbf{1 6}^{11} \mathbf{- 1 8}$ have in their IR spectra two bands, which correspond to sydnone carbonyls and ester carbonyls, in the range $1772-1759$ and $1732-1726 \mathrm{~cm}^{-1}$. In their ${ }^{13} \mathrm{C}$ NMR spectra the signals of the two carbon atoms in the carbonyl groups are observed at 167-169 ppm and the sydnone ring carbon (C-4) appears at 107-109 ppm. The hydroxymethylphenyl derivatives 19-21 have not been analyzed spectroscopically but their oxidation products 22-24 have. The spectroscopic data for 22 correspond to those in the literature ${ }^{12}$ and formylphenylsydnone derivatives $\mathbf{2 3}$ and $\mathbf{2 4}$ show singlets at ca $9.9 \mathrm{ppm}$ in their ${ }^{1} \mathrm{H}$ NMR spectra, which correspond to aldehyde protons, and the characteristic signals of the sydnone carbon (C-4) appear in their ${ }^{13} \mathrm{C}$ NMR spectra at $108-110$ ppm.

The stilbene-sydnone derivatives 1-12, show, besides the characteristic signals for the carbonyl carbon and sydnone ring carbon, the corresponding signals for stilbene moieties in their ${ }^{13} \mathrm{C}$ NMR spectra. All carbonyl carbons and sydnone ring carbons of the cis-and trans-isomers appear in a very narrow region at 167-169 ppm and 97-109 ppm, respectively. The cis-1-12 and trans-1-12 isomers are easily recognizable in their ${ }^{1} \mathrm{H}$ NMR spectra by the coupling constants of the ethylenic protons which have values of $\sim 12 \mathrm{~Hz}$ or $\sim 16 \mathrm{~Hz}$, respectively. In the cases of only 3-substituted sydnone derivatives 1-4 the characteristic singlets for the sydnone ring hydrogens at $\sim 6.5 \mathrm{ppm}$ for the trans-isomers and $6.4 \mathrm{ppm}$ for cis-isomers unequivocally confirm the sydnone structures. 


\section{Biological evaluation}

Stilbene-sydnones were screened for their antiproliferative activity in the Laboratory for Experimental Therapy at the Department for Molecular Medicine of Rudjer Bošković Institute. In vitro studies on 5 cell lines derived from 5 different tumor types: HeLa (cervical carcinoma), MCF-7 (breast carcinoma), SW 620 (colon carcinoma), MiaPaCa-2 (pancreatic carcinoma), and H 460 (lung carcinoma).

The tested compounds showed diverse, but overall very low, antiproliferative effects on the tested cell lines. Interestingly, although many trans-stilbenes, including resveratrol, pterostilbene, piceatannol etc. exhibit high levels of biological activities, ${ }^{13}$ in our study transderivatives showed generally lower activity compared to cis-derivatives. Among the latter, the most interesting compounds are cis-5 and cis-10 which showed significantly more pronounced inhibitory effects towards all cell lines. Such activity can be correlated to the resveratrol activity in tumor cells. ${ }^{14}$ Only cis-2, $c i s-6$ and cis-7 did not show growth inhibition, while from tested trans-derivatives only trans $\mathbf{- 3}$ and trans-6 showed low inhibition of cell growth.

\section{Conclusions}

We have synthesized cis- and trans-stilbene-sydnones 1-12 using the strategy in which the properly substituted sydnone moiety was made first followed by building the stilbene moiety. This approach is especially convenient in the cases where both stilbene diastereomers are the target molecules.

Tested compounds showed diverse antiproliferative effects on tested cell lines that ranged from low to moderate, except cis-5 and cis-10, which showed significantly stronger inhibitory effects towards all cell lines. In general cis-derivatives showed significantly better antiproliferative activity, compared to trans-derivatives, whereby only trans-3 and trans-6 showed low inhibition of cell growth.

\section{Experimental Section}

General. The ${ }^{1} \mathrm{H}$ and ${ }^{13} \mathrm{C}$ NMR spectra were recorded on a Bruker AV-600 Spectrometer at 300 and $600 \mathrm{MHz}$. All NMR spectra were measured in $\mathrm{CDCl}_{3}$ or DMSO using tetramethylsilane as reference. UV spectra were measured on a Varian Cary 50 UV/VIS Spectrophotometer. IR spectra were recorded on FTIR-ATR Vertex 70 Bruker or Perkin-Elmer M-297 spectrophotometer. Mass spectra were obtained on Extrel FT MS 2001 DD, Auto Spec Q (VG Analytical Manchester, GB), on Platform LCZ (Micromass, UK) and/or on a Varian Saturn 2200 equipped with Factor Four Capillary Column VF-5ms. Melting points were obtained using an Original Kofler Mikroheitztisch apparatus (Reichert, Wien). Elemental analyses were carried out on a Perkin-Elmer, Series II, CHNS Analyzer 2400 at Rudjer Bošković Institute. Silica gel 
(Merck $0.063 \mathrm{e} 0.2 \mathrm{~mm}$ ) was used for chromatographic purifications. Thin layer chromatography (TLC) was performed on Merck precoated silica gel 60 F254 plates. Solvents were purified by distillation.

\section{$N$-[2-(Methyloxycarbonyl)phenyl]glycine $(13)^{15}$}

A mixture of methyl anthranilate $(2.4 \mathrm{~g}, 15.9 \mathrm{mmol})$, chloroacetic acid $(1.0 \mathrm{~g}, 10.6 \mathrm{mmol})$ and sodium acetate trihydrate $(1.4 \mathrm{~g}, 10.6 \mathrm{mmol})$ in water $(10 \mathrm{~mL})$, was heated for 6 hours at reflux. The reaction mixture was cooled to $0^{\circ} \mathrm{C}$ (on ice bath), then an aqueous solution of $\mathrm{NaOH}(10 \%)$ was added until $\mathrm{pH} 8$ was reached. After extraction with dichloromethane $(3 \times 30 \mathrm{~mL})$ the water phase was acidified with hydrochloric acid to $\mathrm{pH} 3.5$ resulting in a white solid precipitate. Filtration and desiccation afforded the title compound as a white solid (621 mg, 28\%).

\section{2-Methyl-2-[N-[2-(methyloxycarbonyl)phenyl]amino]acetic acid (14)}

A mixture of methyl anthranilate $(5.9 \mathrm{~g}, 39.0 \mathrm{mmol}), 2$-bromopropionic acid $(4.0 \mathrm{~g}, 26.0 \mathrm{mmol})$ and anhydrous sodium acetate $(2.1 \mathrm{~g}, 26.0 \mathrm{mmol})$ in absolute ethanol $(30 \mathrm{~mL})$ was refluxed for 18 hours. After cooling and evaporation water was added $(40 \mathrm{~mL})$ and the mixture was cooled to $0^{\circ} \mathrm{C}$. An aqueous solution of $\mathrm{NaOH}(10 \%)$ was added dropwise until $\mathrm{pH} 8$ was reached. After extraction with dichloromethane $(3 \times 30 \mathrm{~mL})$ the water phase was acidified with hydrochloric acid to $\mathrm{pH} 3.5$ resulting in precipitation of a white solid. Filtration and desiccation afforded the title compound 14 as a white solid (1.97 g, 34\%).

14. White solid, yield $34 \%, \mathrm{mp} 122-123^{\circ} \mathrm{C}$; UV (EtOH) $\lambda_{\max } / \mathrm{nm}\left(\varepsilon / \mathrm{dm}^{3} \mathrm{~mol}^{-1} \mathrm{~cm}^{-1}\right): 221(29$ 179), 255 (9 806), 348 (6 379). IR (KBr) $v_{\max } / \mathrm{cm}^{-1}: \sim 3300-2500$ (assoc. COOH), 3352 (NH), 1702 (CO), 1685 (CO). ${ }^{1} \mathrm{H}$ NMR $\left(300 \mathrm{MHz},\left(\mathrm{CD}_{3}\right)_{2} \mathrm{CO}\right) \delta / \mathrm{ppm}: 8.19$ (bs, 1H, NH), 7.89 (d, 1H, $J=8.0 \mathrm{~Hz}), 7.39(\mathrm{t}, 1 \mathrm{H}, J=8.0 \mathrm{~Hz}), 6.73(\mathrm{~d}, 1 \mathrm{H}, J=8.0 \mathrm{~Hz}), 6.64(\mathrm{t}, 1 \mathrm{H}, J=8.0 \mathrm{~Hz}), 4.30(\mathrm{q}$, $1 \mathrm{H}, J=7.2 \mathrm{~Hz}, \mathrm{CH}), 3.85(\mathrm{~s}, 3 \mathrm{H}), 1.54\left(\mathrm{~d}, 3 \mathrm{H}, J=7.2 \mathrm{~Hz}, \mathrm{CH}_{3}\right) .{ }^{13} \mathrm{C} \mathrm{NMR}(75 \mathrm{MHz}$, $\left(\mathrm{CD}_{3}\right)_{2} \mathrm{CO}$,) $\delta / \mathrm{ppm}: 175.1$ (s), 166.3 (s), 150.7 (s), 135.5 (d), 132.4 (d), 116.1 (d), 112.6 (d), 111.4 (s), 51.9 (d), 51.4 (q), 19.0 (q). MS, $m / z: 224\left(\mathrm{M}^{+}+1,100 \%\right)$.

\section{2-Phenyl-2- $N$-[2-(methyoxycarbonyl)phenyl]aminoacetic acid (15)}

Mixture of methyl anthranilate $(2.3 \mathrm{~g}, 15.0 \mathrm{mmol}), \alpha$-bromophenylacetic acid (2.15 g, 10.0 $\mathrm{mmol})$ and anhydrous sodium acetate $(820 \mathrm{mg}, 10.0 \mathrm{mmol})$ in absolute ethanol $(35 \mathrm{~mL})$ was refluxed for 6 hours. After cooling and evaporation water was added $(60 \mathrm{~mL})$ and the mixture was cooled to $0^{\circ} \mathrm{C}$. An Aqueous solution of $\mathrm{NaOH}(10 \%)$ was added dropwise until $\mathrm{pH} 8$ was reached. After extraction with dichloromethane $(3 \times 30 \mathrm{~mL})$ the water phase was acidified with hydrochloric acid to $\mathrm{pH} 3.5$ resulting in precipitation of a white solid. Filtration and desiccation afforded the title compound $\mathbf{1 5}$ as a white solid (970 $\mathrm{mg}, 34 \%)$.

15. White solid, yield $34 \%, \mathrm{mp} 148-149^{\circ} \mathrm{C}$; UV (EtOH) $\lambda_{\max } / \mathrm{nm}\left(\varepsilon / \mathrm{dm}^{3} \mathrm{~mol}^{-1} \mathrm{~cm}^{-1}\right): 222(27$ 213), 256 (9 561), 348 (5 926). IR (KBr) $v_{\max } / \mathrm{cm}^{-1}:$ 3300-2500 (assoc. COOH), 3354 (NH), 3029, $2955(\mathrm{CH}), 1694,1667(\mathrm{CO}) .{ }^{1} \mathrm{H}$ NMR (300 MHz, $\left.\left(\mathrm{CD}_{3}\right)_{2} \mathrm{CO}\right) \delta / \mathrm{ppm}: 8.98(\mathrm{~d}, 1 \mathrm{H}, J=5.6$ $\mathrm{Hz}, \mathrm{NH}), 7.89(\mathrm{~d}, 1 \mathrm{H}, J=7.8 \mathrm{~Hz}), 7.58(\mathrm{~d}, 2 \mathrm{H}, J=8.4 \mathrm{~Hz}), 7.42-7.29(\mathrm{~m}, 3 \mathrm{H}), 7.25(\mathrm{t}, 1 \mathrm{H}, J=$ 
$7.8 \mathrm{~Hz}), 6.60(\mathrm{t}, 1 \mathrm{H}, J=7.8 \mathrm{~Hz}), 6.58(\mathrm{~d}, 1 \mathrm{H}, J=7.8 \mathrm{~Hz}), 5.30(\mathrm{~d}, 1 \mathrm{H}, J=5.6 \mathrm{~Hz}, \mathrm{CH}), 3.88(\mathrm{~s}$, $\left.3 \mathrm{H}, \mathrm{OCH}_{3}\right) .{ }^{13} \mathrm{C} \mathrm{NMR}\left(150 \mathrm{MHz},\left(\mathrm{CD}_{3}\right)_{2} \mathrm{CO}\right) \delta / \mathrm{ppm}$ : 172.9 (s), 169.8 (s), 150.3 (s), 139.8 (s), 135.8 (d), 132.8 (d), 130.2 (d), 129.6 (d), 128.7 (d), 116.8 (d), 114.0 (d), 112.3 (s), 60.8 (d), 52.5 (q). MS, $m / z: 285.99\left(\mathrm{M}^{+}, 40 \%\right)$.

\section{Synthesis of ester sydnone derivatives (16) ${ }^{11}$ and (17)}

To a suspension of the corresponding glycine 13 or $14(9.2 \mathrm{mmol})$ in water $(30 \mathrm{~mL})$ cooled on an ice bath, was added hydrochloric acid $(17 \%, 9.2 \mathrm{mmol})$ dropwise and the reaction mixture was stirred for 3 hours. Then a solution of sodium nitrite $(952 \mathrm{mg}, 13.8 \mathrm{mmol})$ in water (5 mL) was added dropwise during 2 hours with cooling on the ice bath. The reaction mixture was stirred for an additional one hour then dichloromethane was added and stirring was continued for an additional 30 minutes. After extraction, drying on anhydrous $\mathrm{MgSO}_{4}$, filtration and evaporation, the nitroso-derivative was isolated. Without any further purification the nitroso-product was dissolved in quintuple the amount of acetic anhydride and the reaction mixture was put in a dark place for 7 days. Then the reaction mixture was poured onto cold water $(100 \mathrm{~mL})$ with cooling and vigorous stirring. Neutralization with sodium bicarbonate, extraction with dichloromethane $(3 \times 30 \mathrm{~mL})$, drying, filtration and evaporation afford the crude sydnone derivative.

3-[2-(Methyloxycarbonyl)phenyl]sydnone (16). ${ }^{11}$ From crude mixture isolated by column chromatography on silica-gel as adsorbent and dichloromethane as eluent. Pale yellow crystals, yield 46\%, $940 \mathrm{mg}$, mp $102-104^{\circ} \mathrm{C}$ (lit. $.^{11} 104-106^{\circ} \mathrm{C}$ ).

4-Methyl-3-[2-(methyloxycarbonyl)phenyl]sydnone (17). From crude mixture isolated by column chromatography on silica-gel as adsorbent and ethyl acetate/petroleum ether (17\%) as eluent. Pale yellow crystals, yield $59 \%, 1.27 \mathrm{~g}, \mathrm{mp} 102-103{ }^{\circ} \mathrm{C}$ (water); UV (EtOH) $\lambda_{\max } / \mathrm{nm}$ $\left(\varepsilon / \mathrm{dm}^{3} \mathrm{~mol}^{-1} \mathrm{~cm}^{-1}\right): 202$ (23 122), 310 (7 150). IR (KBr) $v_{\max } / \mathrm{cm}^{-1}: 3077,3048,2958(\mathrm{CH}), 1772$ (CO), 1732 (CO). ${ }^{1} \mathrm{H}$ NMR (300 MHz, $\mathrm{CDCl}_{3}$ ) $\delta / \mathrm{ppm}: 8.23$ (d, 1H, J=9.2 Hz), 7.85-7.77 (m, $2 \mathrm{H}), 7.47(\mathrm{~d}, 1 \mathrm{H}, J=9.2 \mathrm{~Hz}), 3.83\left(\mathrm{~s}, 3 \mathrm{H}, \mathrm{OCH}_{3}\right), 1.99\left(\mathrm{~s}, 3 \mathrm{H}, \mathrm{CH}_{3}\right) .{ }^{13} \mathrm{C} \mathrm{NMR}(75 \mathrm{MHz}$, $\mathrm{CDCl}_{3}$ ) $\delta /$ ppm: 168.5 (s), 163.5 (s), 133.8 (d), 132.7 (s), 132.5 (d), 132.3 (d), 127.5 (d), 127.3 (s), 107.2 (s), 53.1 (q), 7.4 (q). MS, m/z: $235\left(\mathrm{M}^{+}+1,100 \%\right)$; Elemental analysis, calcd for $\mathrm{C}_{11} \mathrm{H}_{10} \mathrm{~N}_{2} \mathrm{O}_{4}(\mathrm{Mr}=234.21)$ : C 56.41, $\mathrm{H} 4.30, \mathrm{~N} 11.96 \%$; found $\mathrm{C} 56.57, \mathrm{H} 4.25, \mathrm{~N} 12.11 \%$.

\section{4-Phenyl-3-[2-(methyloxycarbonyl)phenyl]sydnone (18)}

To a suspension of $15(2.2 \mathrm{~g}, 7.0 \mathrm{mmol})$ in water $(50 \mathrm{~mL})$ was added with cooling and stirring a solution of sodium nitrite $(2.13 \mathrm{~g}, 30.8 \mathrm{mmol})$ in water $(5 \mathrm{~mL})$ dropwise and the reaction mixture was stirred for 3 hours. Then, $\mathrm{HCl}(17 \%)$ was added dropwise during 3 hours until pH 2 was reached. After an additional hour of stirring on the ice bath, dichloromethane was added (30 $\mathrm{mL}$ ). Extraction, drying, filtration and evaporation afford the crude nitroso product which was dissolved in quintuple the amount of acetic anhydride and the reaction mixture was put in a dark place for 7 days. Then the reaction mixture was poured onto cold water $(150 \mathrm{~mL})$ with cooling and vigorous stirring. Neutralization with sodium bicarbonate, extraction with dichloromethane $(3 \times 30 \mathrm{~mL})$, drying, filtration and evaporation afford the crude sydnone derivative which was 
purified by column chromatography on silica-gel as adsorbent and petroleum ether/ether (1540\%) as eluent giving sydnone 18.

18. Yellow crystals, yield $21 \%, 435 \mathrm{mg}, \mathrm{mp} 160^{\circ} \mathrm{C}$; UV (EtOH) $\lambda_{\max } / \mathrm{nm}\left(\varepsilon / \mathrm{dm}^{3} \mathrm{~mol}^{-1} \mathrm{~cm}^{-1}\right): 202$ (21 817), 338 (6 853). IR (KBr) $v_{\max } / \mathrm{cm}^{-1}: 2954,2846$ (CH), 1759 (CO), 1726 (CO); ${ }^{1} \mathrm{H}$ NMR (300 MHz, $\left.\mathrm{CDCl}_{3}\right) \delta / \mathrm{ppm}: 8.17(\mathrm{~d}, 1 \mathrm{H}, J=9.2 \mathrm{~Hz}), 7.80-7.74(\mathrm{~m}, 2 \mathrm{H}), 7.49(\mathrm{~d}, 1 \mathrm{H}, J=9.2 \mathrm{~Hz})$, 7.26 (bs, 5H), 3.75 (s, 3H, $\left.\mathrm{OCH}_{3}\right) .{ }^{13} \mathrm{C} \mathrm{NMR}\left(75 \mathrm{MHz}, \mathrm{CDCl}_{3}\right) \delta / \mathrm{ppm}: 167.0$ (s, CO-syd), 163.8 (s, CO-est), 133.9 (d), 133.9 (s), 132.6 (d), 132.4 (d), 129.0 (2d), 129.0 (d), 127.9 (s), 127.7 (d), 126.9 (2d), 124.6 (s), 109.6 (s, C-syd), 53.3 (q, CH3-est). MS, m/z: 297 (M+1, 100\%); Elemental analysis, calcd for $\mathrm{C}_{16} \mathrm{H}_{12} \mathrm{~N}_{2} \mathrm{O}_{4}(\mathrm{Mr}=296.28): \mathrm{C} \mathrm{64.86,} \mathrm{H} 4.08, \mathrm{~N} \mathrm{9.46 \%}$; found $\mathrm{C}$ 64.98, H 4.19, N 9.59\%.

\section{Synthesis of alcohol sydnone derivatives (19-21)}

The reaction mixture of the corresponding sydnone 16-18 $(1.9 \mathrm{mmol})$ and $\mathrm{NaBH}_{4}(3.8 \mathrm{mmol})$ in $t$-BuOH $(12 \mathrm{~mL})$ was heated to reflux and $\mathrm{MeOH}(1 \mathrm{~mL})$ was added dropwise during 1 hour. After cooling to room temperature, water $(15 \mathrm{~mL})$ was added and the mixture was evaporated under reduced pressure to remove $t-\mathrm{BuOH} / \mathrm{MeOH}$ as an azeotrope. The product was extracted from water with dichloromethane $(3 \times 25 \mathrm{~mL})$. The organic phase was dried over anhydrous $\mathrm{MgSO}_{4}$, filtered and evaporated to afford the crude product which was used in the next reaction without any further purification 19 (oily matter, yield 59\%, $217 \mathrm{mg}$ ), $\mathbf{2 0}$ (oily matter, yield 58\%, $395 \mathrm{mg}$ ), 21 (oily matter, yield 93\%, $478 \mathrm{mg}$ ).

\section{Synthesis of aldehyde sydnone derivatives $(22),{ }^{12}(23)$ and (24)}

The corresponding alcohol (19-21, $1.3 \mathrm{mmol})$ was dissolved in dichloromethane $(15 \mathrm{~mL})$, then $\mathrm{MnO}_{2}$ (10 eq) was added and the reaction mixture was stirred at room temperature for three days with portionwise addition of additional amounts of $\mathrm{MnO}_{2}$ (20 eq). The product was isolated by column chromatography with silica-gel as adsorbent and with dichloromethane/ether (0-3\%) as eluent.

3-(2-Formylphenyl)sydnone (22). White solid, yield $68 \%, 170 \mathrm{mg}, \quad \mathrm{mp} \quad 93-94{ }^{\circ} \mathrm{C}$ (lit. $\left.{ }^{12} 93-94{ }^{\circ} \mathrm{C}\right)$.

3-(2-Formylphenyl)-4-methylsydnone (23). Oil, yield 61\%, $163 \mathrm{mg}$; IR (neat) $v_{\mathrm{max}} / \mathrm{cm}^{-1}: 3072$,

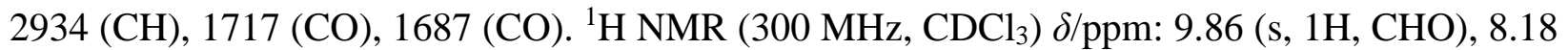
$(\mathrm{d}, 1 \mathrm{H}, J=9.1 \mathrm{~Hz}), 7.95-7.89(\mathrm{~m}, 2 \mathrm{H}), 7.56(\mathrm{~d}, 1 \mathrm{H}, J=9.1 \mathrm{~Hz}), 2.03\left(\mathrm{~s}, 3 \mathrm{H}, \mathrm{CH}_{3}\right) .{ }^{13} \mathrm{C} \mathrm{NMR}(75$ $\mathrm{MHz} \mathrm{CDCl}_{3}$ ) $\delta / \mathrm{ppm}: 186.7$ (d, CHO), 171.3 (s, CO-syd), 135.2 (d), 133.3 (s), 133.0 (d), 131.9 (d), 130.7 (s), 127.2 (d), 107.7 (s, C-syd), 7.6 (q). MS, m/z: $205\left(\mathrm{M}^{+}+1,100 \%\right)$.

4-Phenyl-3-(2-formylphenyl)sydnone (24). Yellow crystals, yield 64\%, $222 \mathrm{mg}, \mathrm{mp} 133^{\circ} \mathrm{C}$; UV (EtOH) $\lambda_{\max } / \mathrm{nm}\left(\varepsilon / \mathrm{dm}^{3} \mathrm{~mol}^{-1} \mathrm{~cm}^{-1}\right): 204$ (20 042), 245 (8 464), 334 (8 926). IR (KBr) $\nu_{\max } / \mathrm{cm}^{-1}:$ 3065, 2953, $2858(\mathrm{CH}), 1739(\mathrm{CO}), 1704(\mathrm{CO}) .{ }^{1} \mathrm{H}$ NMR (300 MHz, CDCl 3 ) $\delta / \mathrm{ppm}$ : $9.86(\mathrm{~s}, 1 \mathrm{H}, \mathrm{CHO}), 8.13(\mathrm{~d}, 1 \mathrm{H}, J=9.1 \mathrm{~Hz}), 7.93-7.85(\mathrm{~m}, 2 \mathrm{H}), 7.60(\mathrm{~d}, 1 \mathrm{H}, J=9.1 \mathrm{~Hz}), 7.27-$ $7.23(\mathrm{~m}, 5 \mathrm{H}) .{ }^{13} \mathrm{C} \mathrm{NMR}\left(75 \mathrm{MHz}, \mathrm{CDCl}_{3}\right.$,) $\delta / \mathrm{ppm}$ : 186.5 (d, CHO), 166.7 (s, CO-syd), 135.5 (d), 
134.5 (s), 133.1 (d), 132.0 (d), 130.8 (s), 129.2 (d), 129.1 (2d), 127.7 (d), 126.9 (2d), 124.1 (s), 110.3 (s, C-syd). MS, m/z: $266.99\left(\mathrm{M}^{+}, 100 \%\right)$.

General procedure for the synthesis of 4-methyl, 4-chloro, 4-bromo and 4methoxybenzylbromides (25-28)

A mixture of $p$-xylene, $p$-chlorotoluene, $p$-bromotoluene or $p$-methylanisole $(0.1 \mathrm{~mol}), \mathrm{CCl}_{4}(100$ $\mathrm{mL}), N$-bromosuccinimide $(19.6 \mathrm{~g}, 0.11 \mathrm{~mol})$ and azoisobutyronitrile $(0.6 \mathrm{~g})$ was heated at reflux for 40 minutes. After succinimide filtration, washing with $\mathrm{CCl}_{4}(40 \mathrm{~mL})$ and solvent evaporation the crude product was isolated and used in the next reaction without any further purification.

General procedure for the synthesis of 4-methyl, 4-chloro, 4-bromo and 4methoxybenzyltriphenylphosphonium bromides (29-32)

The corresponding 4-substituted benzylbromide 25-28 ( $0.1 \mathrm{~mol}$,) was dissolved in toluene (40 $\mathrm{mL})$ and a solution of triphenylphosphine $(26.2 \mathrm{~g}, 0.1 \mathrm{~mol})$ in toluene $(50 \mathrm{~mL})$ was added. The reaction mixture was stirred at room temperature overnight. Filtration, washing with petroleum ether $(5 \mathrm{~mL})$ and drying afford the corresponding $p$-substituted-benzyltriphenylphosphonium bromides 29 (85\%), 30 (73\%), 31 (60\%) 32 (75\%).

\section{General procedure for the synthesis of stilbenylsydnones (1-12)}

The previously prepared $p$-substituted benzyltriphenylphosphonium bromide 29-32 (1.6 mmol) was dissolved in absolute ethanol $(10 \mathrm{~mL})$ under argon and a solution of sodium ethoxide in ethanol, prepared by reaction of $\mathrm{Na}(36 \mathrm{mg}, 1.6 \mathrm{mmol})$ in absolute ethanol $(5 \mathrm{~mL})$, was added dropwise. The reaction mixture was stirred for 15 minutes and a solution of the corresponding aldehyde 22-24 (1.0 mmol in $15 \mathrm{~mL}$ of ethanol) was added. The reaction mixture was stirred and heated at $40{ }^{\circ} \mathrm{C}$ for 1 hour. After solvent evaporation, water $(20 \mathrm{~mL})$ and benzene $(15 \mathrm{~mL})$ were added to the residue. The water phase was washed with benzene $(3 \times 15 \mathrm{~mL})$, the organic layers were collected, dried over anhydrous $\mathrm{MgSO}_{4}$, filtrated and evaporated, affording crude product. The mixtures of the trans- and cis-stilbenylsydnones were separated by column chromatography on silica-gel as adsorbent and dichloromethane as eluent. Further column chromatography with dichloromethane and/or dichloromethane/ether (0-3\%) afforded the pure trans- and pure cisisomers.

3-[2-[2-(4-Methylphenyl)ethenyl]phenyl]sydnone (1). Yield 62\%, $172 \mathrm{mg}$. According to ${ }^{1} \mathrm{H}$ NMR spectrum cis/trans $=1 / 1.2$. Dichloromethane/ether $(0-3 \%)$ used as eluent. Spectroscopic data of trans- and cis-1 correspond to data in the lit. ${ }^{10}$.

3-[2-[2-(4-Chlorophenyl)ethenyl]phenyl]sydnone (2). Yield 77\%, $230 \mathrm{mg}$. According to ${ }^{1} \mathrm{H}$ NMR spectrum cis/trans $=1 / 1$. Dichloromethane/ether (0-3\%) used as eluent.

trans-2. Pale yellow solid, mp $189-190^{\circ} \mathrm{C}$; UV (EtOH) $\lambda_{\max } / \mathrm{nm}\left(\varepsilon / \mathrm{dm}^{3} \mathrm{~mol}^{-1} \mathrm{~cm}^{-1}\right): 203(25$ 306), 223 (17 090), 302 (29 845). IR (diamond) $v_{\max } / \mathrm{cm}^{-1}: 3118$ (CH-syd), 1745 (CO-syd). ${ }^{1} \mathrm{H}$ NMR $\left(600 \mathrm{MHz}, \mathrm{CDCl}_{3}\right) \delta / \mathrm{ppm}: 7.88(\mathrm{~d}, 1 \mathrm{H}, J=7.9 \mathrm{~Hz}), 7.69-7.66(\mathrm{~m}, 1 \mathrm{H}), 7.51-7.50(\mathrm{~m}, 2 \mathrm{H})$, $7.38(\mathrm{~d}, 2 \mathrm{H}, J=8.3 \mathrm{~Hz}, \mathrm{H}-10 / 11), 7.34$ (d, 2H, $J=8.3 \mathrm{~Hz}, \mathrm{H}-10 / 11), 7.15$ (d, 1H, $J=16.1 \mathrm{~Hz}$, 
$\mathrm{H}-7 / 8), 6.83$ (d, 1H, J = 16.1 Hz, H-7/8), 6.55 (s, 1H, H-syd). ${ }^{13} \mathrm{C}$ NMR (150 MHz, CDCl 3 )

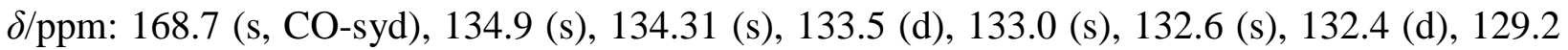
(d), 128.8 (d), 128.3 (d), 127.3 (d), 125.6 (d), 120.5 (d), 98.0 (d, CH-syd). MS, m/z: 298.91 (M+, $100 \%), 300.89\left(\mathrm{M}^{+}, 45 \%\right)$; Elemental analysis, calcd for $\mathrm{C}_{16} \mathrm{H}_{11} \mathrm{ClN}_{2} \mathrm{O}_{2}(\mathrm{Mr}=298.72)$ : $\mathrm{C} 64.33$, H 3.71, N 9.38\%; found C 64.05, H 3.79, N 9.54\%.

cis-2. Pale yellow solid, mp 124-125 ${ }^{\circ} \mathrm{C}$; UV (EtOH) $\lambda_{\max } / \mathrm{nm}\left(\varepsilon / \mathrm{dm}^{3} \mathrm{~mol}^{-1} \mathrm{~cm}^{-1}\right): 203$ (39 790), 264 (16 691), 290 (16 280). IR (diamond) $v_{\max } / \mathrm{cm}^{-1}: 3128$ (CH-syd), 1720 (CO-syd). ${ }^{1} \mathrm{H}$ NMR $\left(300 \mathrm{MHz}, \mathrm{CDCl}_{3}\right) \delta / \mathrm{ppm}: 7.56-7.49(\mathrm{~m}, 3 \mathrm{H}), 7.42(\mathrm{~d}, 1 \mathrm{H}, J=8.0 \mathrm{~Hz}), 7.19$ (d, 2H, J=8.6 Hz, $\mathrm{H}-10 / 11), 7.00$ (d, 2H, $J=8.6 \mathrm{~Hz}, \mathrm{H}-10 / 11), 6.73$ (d, 1H, $J=12.0 \mathrm{~Hz}, \mathrm{H}-7 / 8), 6.45$ (d, 1H, $J=$ $12.0 \mathrm{~Hz}, \mathrm{H}-7 / 8), 6.46$ (s, 1H, H-syd). ${ }^{13} \mathrm{C} \mathrm{NMR}\left(150 \mathrm{MHz}, \mathrm{CDCl}_{3}\right.$ ) $\delta / \mathrm{ppm}: 168.8$ (s, CO-syd), 134.1 (s), 133.9 (d), 133.3 (s), 132.9 (s, 2C), 132.3 (d), 131.6 (d), 130.2 (d), 129.2 (d), 128.9 (d), 125.4 (d), 123.6 (d), 97.3 (d, CH-syd). MS, m/z: $298.91\left(\mathrm{M}^{+}, 100 \%\right), 300.89\left(\mathrm{M}^{+}, 45 \%\right)$.

3-[2-[2-(4-Bromophenyl)ethenyl]phenyl]sydnone (3). Yield 72\%, $247 \mathrm{mg}$. According to ${ }^{1} \mathrm{H}$ NMR spectrum cis/trans $=1.3 / 1$. Dichloromethane/ether was $(0-3 \%)$ used as eluent.

trans-3. Yellow solid, mp 191-192 ${ }^{\circ} \mathrm{C}$; UV (EtOH) $\lambda_{\max } / \mathrm{nm}\left(\varepsilon / \mathrm{dm}^{3} \mathrm{~mol}^{-1} \mathrm{~cm}^{-1}\right)$ : 202 (22 748), 226 (15 815), 302 (31 794). IR (diamond) $v_{\max } / \mathrm{cm}^{-1}: 3113$ (CH-syd), 1732 (CO, syd). ${ }^{1} \mathrm{H}$ NMR $\left(600 \mathrm{MHz}, \mathrm{CDCl}_{3}\right) \delta / \mathrm{ppm}: 7.88(\mathrm{~d}, 1 \mathrm{H}, J=8.0 \mathrm{~Hz}), 7.69-7.66(\mathrm{~m}, 1 \mathrm{H}), 7.51-7.49(\mathrm{~m}, 4 \mathrm{H}), 7.31$ (d, 2H, $J=8.4 \mathrm{~Hz}, \mathrm{H}-10 / 11), 7.13$ (d, 1H, $J=16.2 \mathrm{~Hz}, \mathrm{H}-7 / 8), 6.84$ (d, 1H, $J=16.2 \mathrm{~Hz}, \mathrm{H}-7 / 8)$, 6.55 (s, 1H, H-syd). ${ }^{13} \mathrm{C}$ NMR (150 MHz, CDCl 3 ) $\delta / \mathrm{ppm}: 168.9$ (s, CO-syd), 134.9 (s), 133.7 (d), 133.1 (s), 132.7 (s), 132.6 (d), 132.3 (d), 129.0 (d), 128.7 (d), 127.4 (d), 125.8 (d), 123.3 (s), 120.8 (d), 98.2 (d, CH-syd). MS m/z: $344.81\left(\mathrm{M}^{+}, 100 \%\right), 342.81\left(\mathrm{M}^{+}, 100 \%\right)$. Elemental analysis, calcd for $\mathrm{C}_{16} \mathrm{H}_{11} \mathrm{BrN}_{2} \mathrm{O}_{2}(\mathrm{Mr}=343.11)$ : C 56.00, $\mathrm{H} \mathrm{3.23,} \mathrm{N} \mathrm{8.16 \% ;} \mathrm{found} \mathrm{C} \mathrm{56.24,} \mathrm{H}$ 3.12, N 8.25\%.

cis-3. Yellow solid, mp $151^{\circ} \mathrm{C}$; UV (EtOH) $\lambda_{\max } / \mathrm{nm}\left(\varepsilon / \mathrm{dm}^{3} \mathrm{~mol}^{-1} \mathrm{~cm}^{-1}\right): 203$ (60 792), 219 (44 234), 269 (27 861), 290 (27 357). IR (diamond) $v_{\max } / \mathrm{cm}^{-1}: 3130$ (CH-syd), 1720 (CO-syd). ${ }^{1} \mathrm{H}$ NMR $\left(300 \mathrm{MHz}, \mathrm{CDCl}_{3}\right) \delta / \mathrm{ppm}: 7.55-7.50(\mathrm{~m}, 3 \mathrm{H}), 7.42(\mathrm{~d}, 1 \mathrm{H}, J=8.1 \mathrm{~Hz}), 7.35(\mathrm{~d}, 2 \mathrm{H}, J=$ $8.4 \mathrm{~Hz}, \mathrm{H}-10 / 11$ ), 6.94 (d, 2H, $J=8.4 \mathrm{~Hz}, \mathrm{H}-10 / 11$ ), 6.70 (d, 1H, $J=12.0 \mathrm{~Hz}, \mathrm{H}-7 / 8), 6.46$ (d, $1 \mathrm{H}, J=12.0 \mathrm{~Hz}, \mathrm{H}-7 / 8), 6.44$ (s, 1H, H-syd). ${ }^{13} \mathrm{C} \mathrm{NMR}\left(150 \mathrm{MHz}, \mathrm{CDCl}_{3}\right) \delta / \mathrm{ppm}: 168.9$ (s, COsyd), 134.3 (s), 134.0 (d), 133.3 (s), 132.9 (s), 132.3 (d), 131.9 (d), 131.6 (d), 130.4 (d), 129.2 (d), 125.4 (d), 123.7 (d), 122.4 (s), 97.3 (d, CH-syd). MS, m/z: $344.81\left(\mathrm{M}^{+}, 100 \%\right), 342.81\left(\mathrm{M}^{+}\right.$, $100 \%)$.

3-[2-[2-(4-Methoxyphenyl)ethenyl]phenyl]sydnone (4). Yield 73\%, $215 \mathrm{mg}$. According to ${ }^{1} \mathrm{H}$ NMR spectrum cis/trans $=1 / 1.2$. Dichloromethane/ether was $(4 \%)$ used as eluent.

trans-4. Yellow solid, mp $112-113^{\circ} \mathrm{C}$; UV (EtOH) $\lambda_{\max } / \mathrm{nm}\left(\varepsilon / \mathrm{dm}^{3} \mathrm{~mol}^{-1} \mathrm{~cm}^{-1}\right): 202$ (21 723), 227 (13 623), 317 (24 230). IR (diamond) $v_{\max } / \mathrm{cm}^{-1}: 3111$ (CH-syd), 1764 (CO-syd). ${ }^{1} \mathrm{H}$ NMR $\left(600 \mathrm{MHz}, \mathrm{CDCl}_{3}\right) \delta / \mathrm{ppm}: 7.80(\mathrm{~d}, 1 \mathrm{H}, J=8.1 \mathrm{~Hz}), 7.66-7.63(\mathrm{~m}, 1 \mathrm{H}), 7.48-7.46(\mathrm{~m}, 2 \mathrm{H}), 7.40$ $(\mathrm{d}, 2 \mathrm{H}, J=8.8 \mathrm{~Hz}), 7.17(\mathrm{~d}, 1 \mathrm{H}, J=16.0 \mathrm{~Hz}), 6.92(\mathrm{~d}, 2 \mathrm{H}, J=8.8 \mathrm{~Hz}), 6.72(\mathrm{~d}, 1 \mathrm{H}, J=16.0$ $\mathrm{Hz}$ ), 6.57 (s, 1H, CH-syd), $3.86\left(\mathrm{~s}, 3 \mathrm{H}, \mathrm{OCH}_{3}\right) .{ }^{13} \mathrm{C} \mathrm{NMR}\left(150 \mathrm{MHz}, \mathrm{CDCl}_{3}\right) \delta / \mathrm{ppm}: 169.0$ (s, CO-syd), 160.6 (s), 134.6 (d), 133.8 (s), 132.5 (d), 132.5 (s), 128.8 (s), 128.7 (d), 128.2 (d), 127.2 (d), 125.7 (d), 117.7 (d), 114.6 (d), 98.3 (d, CH-syd), 55.63 (q, $\mathrm{OCH}_{3}$ ). MS, m/z: 294.96 
$\left(\mathrm{M}^{+}, 100 \%\right)$. Elemental analysis, calcd for $\mathrm{C}_{17} \mathrm{H}_{14} \mathrm{~N}_{2} \mathrm{O}_{3}(\mathrm{Mr}=294.3)$ : $\mathrm{C} 69.38, \mathrm{H} 4.79, \mathrm{~N} 9.52 \%$; found C 69.18, H 4.67, N 9.45\%.

cis-4. White solid, mp $111^{\circ} \mathrm{C}$; UV (EtOH) $\lambda_{\max } / \mathrm{nm}\left(\varepsilon / \mathrm{dm}^{3} \mathrm{~mol}^{-1} \mathrm{~cm}^{-1}\right): 203$ (35 218), 292 (15 657). IR (diamond) $v_{\max } / \mathrm{cm}^{-1}: 3120(\mathrm{CH}-\mathrm{syd}), 1728$ (CO-syd). ${ }^{1} \mathrm{H}$ NMR $\left(600 \mathrm{MHz}, \mathrm{CDCl}_{3}\right)$ d/ppm: 7.53-7.46 (m, 4H), $7.00(\mathrm{~d}, 2 \mathrm{H}, J=8.3 \mathrm{~Hz}), 6.73(\mathrm{~d}, 2 \mathrm{H}, J=8.3 \mathrm{~Hz}), 6.70(\mathrm{~d}, 1 \mathrm{H}, J=$ $12.0 \mathrm{~Hz}, \mathrm{H}-7 / 8), 6.42$ (s, 1H, CH-syd), 6.31 (d, $1 \mathrm{H}, J=12.0 \mathrm{~Hz}, \mathrm{H}-7 / 8), 3.78\left(\mathrm{~s}, 3 \mathrm{H}, \mathrm{OCH}_{3}\right) .{ }^{13} \mathrm{C}$

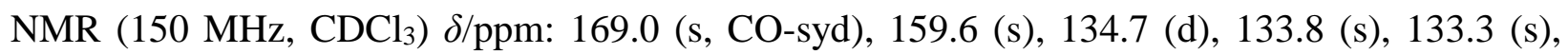
132.2 (d), 131.8 (d), 130.3 (d), 128.8 (d), 127.9 (s), 125.3 (d), 121.0 (d), 114.1 (d), 97.4 (d, CHsyd), $55.4\left(\mathrm{q}, \mathrm{OCH}_{3}\right)$. MS, $m / z: 294.96\left(\mathrm{M}^{+}, 100 \%\right)$.

4-Methyl-3-[2-[2-(4-methylphenyl)ethenyl]phenyl]sydnone (5). Yield $62 \%, 181 \mathrm{mg}$. According to ${ }^{1} \mathrm{H}$ NMR spectrum cis/trans $=1.3 / 1$. Dichloromethane/ether was $(0-3 \%)$ used as eluent.

trans-5. White solid. Spectroscopic data correspond to data in ref. 10a.

cis-5. White solid, mp 105-107 ${ }^{\circ} \mathrm{C}$; UV (EtOH) $\lambda_{\max } / \mathrm{nm}\left(\varepsilon / \mathrm{dm}^{3} \mathrm{~mol}^{-1} \mathrm{~cm}^{-1}\right): 204$ (30 095), 221 (18 600), 297 (13 093). IR (diamond) $v_{\max } / \mathrm{cm}^{-1}: 3021,2921,2857(\mathrm{CH}), 1733$ (CO-syd); ${ }^{1} \mathrm{H}$ NMR $\left(600 \mathrm{MHz}, \mathrm{CDCl}_{3}\right) \delta / \mathrm{ppm}: 7.52(\mathrm{~d}, 1 \mathrm{H}, J=9.0 \mathrm{~Hz}), 7.49-7.47(\mathrm{~m}, 2 \mathrm{H}), 7.40(\mathrm{~d}, 1 \mathrm{H}, J=$ $9.0 \mathrm{~Hz}$ ), 7.03 (s, 4H, H-10 and H-11), 6.68 (d, 1H, $J=12.2 \mathrm{~Hz}, \mathrm{H}-7 / 8), 6.16$ (d, 1H, $J=12.2 \mathrm{~Hz}$, $\mathrm{H}-7 / 8), 2.31$ (s, 3H, CH$\left.)_{3}\right), 1.96\left(\mathrm{~s}, 3 \mathrm{H}, \mathrm{CH}_{3}\right) .{ }^{13} \mathrm{C} \mathrm{NMR}\left(150 \mathrm{MHz}, \mathrm{CDCl}_{3}\right) \delta / \mathrm{ppm}$ : 168.9 (COsyd), 138.4 (s), 135.1 (d), 134.7 (s), 132.6 (s), 132.3 (s), 131.9 (d), 131.5 (d), 129.4 (d), 128.9 (d), 128.9 (d), 126.4 (d), 121.6 (d), 106.7 (s, C-syd), 21.4 (q, $\mathrm{CH}_{3}$ ), 7.6 (q, $\mathrm{CH}_{3}$ ). MS, m/z: 292 $\left(\mathrm{M}^{+}, 100 \%\right), 248$ (11), 207 (60). Elemental analysis, calcd for $\mathrm{C}_{18} \mathrm{H}_{16} \mathrm{~N}_{2} \mathrm{O}_{2}(\mathrm{Mr}=292.12)$ : $\mathrm{C}$ 73.95, H 5.52, N 9.58\%; found C 73.70, H 5.44, N 9.70\%.

3-[2-[2-(4-Chlorophenyl)ethenyl]phenyl]-4-methylsydnone (6). Yield 39\%, $122 \mathrm{mg}$. According to ${ }^{1} \mathrm{H}$ NMR spectrum cis/trans $=1.2 / 1$. Dichloromethane/ether was $(0-3 \%)$ used as eluent.

trans-6. Yellowish solid, mp 191-192 ${ }^{\circ} \mathrm{C}$; UV (EtOH) $\lambda_{\max } / \mathrm{nm}\left(\varepsilon / \mathrm{dm}^{3} \mathrm{~mol}^{-1} \mathrm{~cm}^{-1}\right): 202(22635)$, 228 (14 817), 305 (34 200). IR (diamond) $v_{\max } / \mathrm{cm}^{-1}: 3066(\mathrm{CH}), 1732$ (CO-syd). ${ }^{1} \mathrm{H}$ NMR (600 $\left.\mathrm{MHz}, \mathrm{CDCl}_{3}\right) \delta / \mathrm{ppm}: 7.90(\mathrm{~d}, 1 \mathrm{H}, J=8.2 \mathrm{~Hz}), 7.68(\mathrm{t}, 1 \mathrm{H}, J=8.2 \mathrm{~Hz}), 7.52(\mathrm{t}, 1 \mathrm{H}, J=8.2 \mathrm{~Hz})$, 7.39 (d, 1H, $J=8.2 \mathrm{~Hz}$ ), 7.34 (s, 4H, H-10 and H-11), 7.14 (d, 1H, J = $16.2 \mathrm{~Hz}, \mathrm{H}-7 / 8), 6.60$ (d, $1 \mathrm{H}, J=16.2 \mathrm{~Hz}, \mathrm{H}-7 / 8), 1.96\left(\mathrm{~s}, 3 \mathrm{H}, \mathrm{CH}_{3}\right) .{ }^{13} \mathrm{C} \mathrm{NMR}\left(150 \mathrm{MHz}, \mathrm{CDCl}_{3}\right) \delta / \mathrm{ppm}$ : 168.7 (s, COsyd), 135.0 (s), 134.4 (s), 133.6 (s), 133.5 (d), 132.6 (d), 131.4 (s), 129.3 (d), 129.0 (d), 128.4 (d), 127.1 (d), 126.6 (d), 120.4 (d), 107.1 (s, C-syd), 7.6 (q, $\left.\mathrm{CH}_{3}\right) . \mathrm{MS}, m / z: 312.92\left(\mathrm{M}^{+}, 100 \%\right)$, $314.91\left(\mathrm{M}^{+}, 45 \%\right)$. Elemental analysis, calcd for $\mathrm{C}_{17} \mathrm{H}_{13} \mathrm{ClN}_{2} \mathrm{O}_{2}(\mathrm{Mr}=312.75)$ : C 65.29, $\mathrm{H} 4.19$, N 8.96\%; found C 65.41, H 4.23, N 8.77\%.

cis-6. White solid, mp $148^{\circ} \mathrm{C}$; UV (EtOH) $\lambda_{\max } / \mathrm{nm}\left(\varepsilon / \mathrm{dm}^{3} \mathrm{~mol}^{-1} \mathrm{~cm}^{-1}\right): 202$ (22 244), 222 (22 088), 296 (15 468). IR (diamond) $v_{\max } / \mathrm{cm}^{-1}: 2925$ (CH), 1730 (CO-syd). ${ }^{1} \mathrm{H}$ NMR (600 MHz, $\left.\mathrm{CDCl}_{3}\right) \delta /$ ppm: 7.51-7.50 (m, 2H), $7.46(\mathrm{~d}, 1 \mathrm{H}, J=8.8 \mathrm{~Hz}), 7.41(\mathrm{~d}, 1 \mathrm{H}, J=8.8 \mathrm{~Hz}), 7.20(\mathrm{~d}$, $2 \mathrm{H}, J=8.4 \mathrm{~Hz}), 7.05(\mathrm{~d}, 2 \mathrm{H}, J=8.4 \mathrm{~Hz}), 6.67(\mathrm{~d}, 1 \mathrm{H}, J=12.2 \mathrm{~Hz}, \mathrm{H}-7 / 8), 6.25(\mathrm{~d}, 1 \mathrm{H}, J=12.2$ $\mathrm{Hz}, \mathrm{H}-7 / 8), 1.97$ (s, 3H, CH 3 ). ${ }^{13} \mathrm{C}$ NMR (150 MHz, $\left.\mathrm{CDCl}_{3}\right) \delta / \mathrm{ppm}: 168.7$ (s, CO-syd), 134.3 (s), 
134.1 (s), 134.0 (s), 133.9 (d), 132.4 (s), 132.1 (d), 131.3 (d), 130.2 (d), 129.2 (d), 129.0 (d), 126.5 (d), 123.2 (d), 106.4 (s, C-syd), 7.6 (q). MS, m/z: $312.92\left(\mathrm{M}^{+}, 100 \%\right), 314.91\left(\mathrm{M}^{+}, 45 \%\right)$. 3-[2-[2-(4-Bromophenyl)ethenyl]phenyl]-4-methylsydnone (7). Yield 64\%, $229 \mathrm{mg}$. According to ${ }^{1} \mathrm{H}$ NMR spectrum cis/trans $=0.8 / 1$. Dichloromethane/ether was $(0-3 \%)$ used as eluent.

trans-7. Orange solid, mp 192-193 ${ }^{\circ} \mathrm{C}$; UV (EtOH) $\lambda_{\max } / \mathrm{nm}\left(\varepsilon / \mathrm{dm}^{3} \mathrm{~mol}^{-1} \mathrm{~cm}^{-1}\right): 202$ (25 654), 228 (16 424), 306 (35 995). IR (diamond) $v_{\max } / \mathrm{cm}^{-1}: 2931,2835(\mathrm{CH}), 1734$ (CO-syd); ${ }^{1} \mathrm{H}$ NMR $\left(300 \mathrm{MHz}, \mathrm{CDCl}_{3}\right) \delta / \mathrm{ppm}: 7.90(\mathrm{~d}, 1 \mathrm{H}, J=7.9 \mathrm{~Hz}), 7.69(\mathrm{t}, 1 \mathrm{H}, J=7.9 \mathrm{~Hz}), 7.52(\mathrm{t}, 1 \mathrm{H}, J=7.9$ $\mathrm{Hz}), 7.49(\mathrm{~d}, 2 \mathrm{H}, J=8.4 \mathrm{~Hz}), 7.39(\mathrm{~d}, 1 \mathrm{H}, J=7.9 \mathrm{~Hz}), 7.27(\mathrm{~d}, 2 \mathrm{H}, J=8.4 \mathrm{~Hz}), 7.13(\mathrm{~d}, 1 \mathrm{H}, J$ $=16.2 \mathrm{~Hz}, \mathrm{H}-7 / 8), 6.62(\mathrm{~d}, 1 \mathrm{H}, J=16.2 \mathrm{~Hz}, \mathrm{H}-7 / 8), 1.96\left(\mathrm{~s}, 3 \mathrm{H}, \mathrm{CH}_{3}\right) .{ }^{13} \mathrm{C} \mathrm{NMR}(150 \mathrm{MHz}$, $\mathrm{CDCl}_{3}$ ) $\delta /$ ppm: 168.7 (s, CO-syd), 134.8 (s), 134.8 (s), 133.6 (d), 132.6 (d), 132.2 (d), 131.4 (s), 129.0 (d), 128.6 (d), 127.1 (d), 126.6 (d), 123.2 (s), 120.4 (d), 107.1 (s, C-syd), 7.6 (q). MS, m/z: $356.81\left(\mathrm{M}^{+}, 100 \%\right), 358.81\left(\mathrm{M}^{+}, 100 \%\right)$. Elemental analysis, calcd for $\mathrm{C}_{17} \mathrm{H}_{13} \mathrm{BrN}_{2} \mathrm{O}_{2}(\mathrm{Mr}=$ 357.2): C 57.16, H 3.67, N 7.84\%; found C 57.20, H 3.85, N 7.64\%.

cis-7. Pale brown solid, mp 139-140 ${ }^{\circ} \mathrm{C}$; UV (EtOH) $\lambda_{\max } / \mathrm{nm}\left(\varepsilon / \mathrm{dm}^{3} \mathrm{~mol}^{-1} \mathrm{~cm}^{-1}\right): 202$ (32 832), 221 (20 037), 296 (14 567). IR (diamond) $v_{\max } / \mathrm{cm}^{-1}: 3062(\mathrm{CH}), 1732$ (CO-syd). ${ }^{1} \mathrm{H}$ NMR (300 $\left.\mathrm{MHz} \mathrm{CDCl}_{3}\right) \delta / \mathrm{ppm}$ : 7.52-7.40 (m, 4H), $7.35(\mathrm{~d}, 2 \mathrm{H}, J=8.5 \mathrm{~Hz}), 7.00(\mathrm{~d}, 2 \mathrm{H}, J=8.5 \mathrm{~Hz}), 6.65$ $(\mathrm{d}, 1 \mathrm{H}, J=12.2 \mathrm{~Hz}, \mathrm{H}-7 / 8), 6.27(\mathrm{~d}, 1 \mathrm{H}, J=12.2 \mathrm{~Hz}, \mathrm{H}-7 / 8), 1.97\left(\mathrm{~s}, 3 \mathrm{H}, \mathrm{CH}_{3}\right) .{ }^{13} \mathrm{C} \mathrm{NMR}(75$ $\mathrm{MHz}, \mathrm{CDCl}_{3}$ ) $\delta / \mathrm{ppm}: 168.7$ (s, CO-syd), 134.3 (s), 133.9 (s), 133.9 (d), 132.3 (s), 132.1 (d), 131.9 (d), 131.3 (d), 130.5 (d), 129.2 (d), 126.5 (d), 123.2 (d), 122.4 (s), 106.5 (s, C-syd), 7.6 (q, $\left.\mathrm{CH}_{3}\right)$. MS, $m / z: 356.81\left(\mathrm{M}^{+}, 100 \%\right), 358.81\left(\mathrm{M}^{+}, 100 \%\right)$.

4-Methyl-3-[2-[2-(4-methoxyphenyl)ethenyl]phenyl]sydnone (8). Yield 54\%, $167 \mathrm{mg}$. According to ${ }^{1} \mathrm{H}$ NMR spectrum cis/trans $=0.8 / 1$. Dichloromethane/ether $(4 \%)$ was used as eluent.

trans-8. Orange oil; IR (diamond) $v_{\max } / \mathrm{cm}^{-1}: 2930(\mathrm{CH}), 1724$ (CO-syd). ${ }^{1} \mathrm{H}$ NMR (300 MHz, $\left.\mathrm{CDCl}_{3}\right) \delta / \mathrm{ppm}: 7.89(\mathrm{~d}, 1 \mathrm{H}, J=7.9 \mathrm{~Hz}), 7.66(\mathrm{t}, 1 \mathrm{H}, J=7.9 \mathrm{~Hz}), 7.46(\mathrm{t}, 1 \mathrm{H}, J=7.9 \mathrm{~Hz}), 7.37-$ 7.34 (m, 3H), $7.16(\mathrm{~d}, 1 \mathrm{H}, J=16.0 \mathrm{~Hz}, \mathrm{H}-7 / 8), 6.89$ (d, 2H, $J=8.7 \mathrm{~Hz}), 6.49$ (d, 1H, $J=16.0$ $\mathrm{Hz}, \mathrm{H}-7 / 8), 3.83$ (s, 3H, $\left.\mathrm{OCH}_{3}\right), 1.96$ (s, 3H, $\left.\mathrm{CH}_{3}\right) .{ }^{13} \mathrm{C} \mathrm{NMR} \mathrm{(150} \mathrm{MHz,} \mathrm{CDCl}_{3}$ ) $\delta / \mathrm{ppm}: 168.8$ (s, CO-syd), 160.6 (s), 134.4 (d), 134.3 (s), 132.4 (d), 131.1 (s), 128.7 (s), 128.6 (d), 128.3 (d), 126.7 (d), 126.5 (d), 117.4 (d), 114.5 (d), 107.1 (s, C-syd), 55.6 (q, $\left.\mathrm{OCH}_{3}\right), 7.6$ (q, $\left.\mathrm{CH}_{3}\right) . \mathrm{MS}$, $\mathrm{m} / z: 308.96\left(\mathrm{M}^{+}, 100 \%\right)$.

cis-8. Yellowish oil; IR (diamond) $v_{\max } / \mathrm{cm}^{-1}$ : $2931(\mathrm{CH}), 1724$ (CO-syd). ${ }^{1} \mathrm{H}$ NMR (300 MHz, $\left.\mathrm{CDCl}_{3}\right) \delta /$ ppm: 7.57-7.36 (m, 4H), $7.08(\mathrm{~d}, 2 \mathrm{H}, J=8.7 \mathrm{~Hz}), 6.75(\mathrm{~d}, 2 \mathrm{H}, J=8.7 \mathrm{~Hz}), 6.64(\mathrm{~d}$, $1 \mathrm{H}, J=12.0 \mathrm{~Hz}, \mathrm{H}-7 / 8), 6.11(\mathrm{~d}, 1 \mathrm{H}, J=12.0 \mathrm{~Hz}, \mathrm{H}-7 / 8), 3.79\left(\mathrm{~s}, \mathrm{OCH}_{3}\right), 1.96\left(\mathrm{~s}, 3 \mathrm{H}, \mathrm{CH}_{3}\right)$.

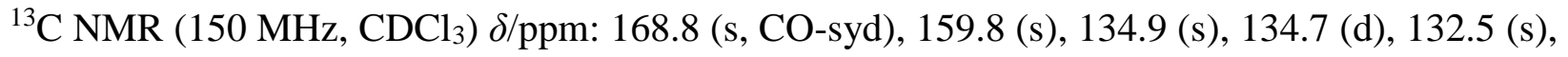
132.0 (d), 131.5 (d), 130.4 (d), 128.8 (d), 128.0 (s), 126.4 (d), 120.6 (d), 114.2 (d), 106.5 (s, Csyd), 55.4 (q, $\left.\mathrm{OCH}_{3}\right), 7.6$ (q). MS, $m / z: 308.96\left(\mathrm{M}^{+}, 100 \%\right)$.

4-Phenyl-3-[2-[2-(4-methylphenyl)ethenyl]phenyl]sydnone (9). Yield 72\%, $255 \mathrm{mg}$. According to ${ }^{1} \mathrm{H}$ NMR spectrum cis/trans $=1 / 1.3$. Dichloromethane was used as eluent. trans-9. White solid. Spectroscopic data correspond to data in ref. 10a. 
cis-9. White solid, mp $127-128^{\circ} \mathrm{C}$; UV (EtOH) $\lambda_{\max } / \mathrm{nm}\left(\varepsilon / \mathrm{dm}^{3} \mathrm{~mol}^{-1} \mathrm{~cm}^{-1}\right): 203$ (42 777), 226 (22 339), 313 (12 603). IR (diamond) $v_{\max } / \mathrm{cm}^{-1}:$ 3060, 3016, $2918(\mathrm{CH}), 1737$ (CO-syd). ${ }^{1} \mathrm{H}$ NMR $\left(300 \mathrm{MHz}, \mathrm{CDCl}_{3}\right) \delta / \mathrm{ppm}: 7.56(\mathrm{~d}, 1 \mathrm{H}, J=8.1 \mathrm{~Hz}), 7.51-7.23(\mathrm{~m}, 8 \mathrm{H}), 6.93(\mathrm{~d}, 2 \mathrm{H}, J=$ $8.0 \mathrm{~Hz}), 6.74(\mathrm{~d}, 2 \mathrm{H}, J=8.0 \mathrm{~Hz}), 6.56$ (d, 1H, $J=12.2 \mathrm{~Hz}, \mathrm{H}-7 / 8), 5.96$ (d, 1H, J = 12.2 Hz, H7/8), 2.28 (s, 3H, $\left.\mathrm{CH}_{3}\right) .{ }^{13} \mathrm{C} \mathrm{NMR}\left(150 \mathrm{MHz} \mathrm{CDCl}_{3}\right.$ ) $\delta / \mathrm{ppm}: 167.0$ (s, CO-syd), 137.9 (s), 135.2 (d), 134.4 (s), 133.2 (s), 132.3 (s), 131.7 (d), 131.4 (d), 129.0 (d), 128.8 (d), 128.7 (d), 128.6 (d), 128.6 (d), 126.7 (d), 126.6 (d), 124.7 (s), 121.3 (d), 109.1 (s, C-syd), 21.2 (q). MS, m/z: 355 $\left(\mathrm{M}^{+}+1,32 \%\right)$. Elemental analysis, calcd for $\mathrm{C}_{23} \mathrm{H}_{18} \mathrm{~N}_{2} \mathrm{O}_{2}(\mathrm{Mr}=354.4): \mathrm{C} 77.95, \mathrm{H} \mathrm{5.12}, \mathrm{N}$ 7.90\%; found C 78.06, H 5.44, N 7.81\%.

4-Phenyl-3-[2-[2-(4-chlorophenyl)ethenyl]phenyl]sydnone (10). Yield 72\%, $270 \mathrm{mg}$. According to ${ }^{1} \mathrm{H}$ NMR spectrum cis/trans $=1 / 0.6$. Dichloromethane was used as eluent.

trans-10. White solid, mp 163-164 ${ }^{\circ} \mathrm{C}$; UV (EtOH) $\lambda_{\max } / \mathrm{nm}\left(\varepsilon / \mathrm{dm}^{3} \mathrm{~mol}^{-1} \mathrm{~cm}^{-1}\right): 202$ (22 244), 230 (11 774), 314 (17 619). IR (diamond) $v_{\max } / \mathrm{cm}^{-1}: 3065(\mathrm{CH}), 1732$ (CO-syd). ${ }^{1} \mathrm{H}$ NMR (300 $\left.\mathrm{MHz}, \mathrm{CDCl}_{3}\right) \delta / \mathrm{ppm}: 7.85(\mathrm{~d}, 1 \mathrm{H}, J=7.7 \mathrm{~Hz}), 7.68(\mathrm{t}, 1 \mathrm{H}, J=7.7 \mathrm{~Hz}), 7.50(\mathrm{t}, 1 \mathrm{H}, J=7.7 \mathrm{~Hz})$, $7.45(\mathrm{~d}, 1 \mathrm{H}, J=7.7 \mathrm{~Hz}), 7.30-7.18(\mathrm{~m}, 9 \mathrm{H}), 6.97(\mathrm{~d}, 1 \mathrm{H}, J=16.3 \mathrm{~Hz}, \mathrm{H}-7 / 8), 6.64(\mathrm{~d}, 1 \mathrm{H}, J=$ 16.3 Hz, H-7/8). ${ }^{13} \mathrm{C}$ NMR (150 MHz, $\mathrm{CDCl}_{3}$ ) $\delta / \mathrm{ppm}: 167.0$ (s, CO-syd), 134.9 (s), 134.5 (s), 133.9 (s), 133.4 (d), 132.6 (d), 132.5 (s), 129.2 (d), 129.2 (d), 129.0 (d), 129.0 (d), 128.3 (d), 127.3 (d), 127.0 (d), 126.5 (d), 124.6 (s), 120.7 (d), 109.6 (s, C-syd). MS, m/z: 374.89 (M', $100 \%), 376.87\left(\mathrm{M}^{+}, 45 \%\right)$. Elemental analysis, calcd for $\mathrm{C}_{22} \mathrm{H}_{15} \mathrm{ClN}_{2} \mathrm{O}_{2}(\mathrm{Mr}=374.82): \mathrm{C} 70.50$, H 4.03, N 7.47\%; found C 70.72, H 4.20, N 7.25\%.

cis-10. White solid, mp $147-148^{\circ} \mathrm{C}$; UV (EtOH) $\lambda_{\max } / \mathrm{nm}\left(\varepsilon / \mathrm{dm}^{3} \mathrm{~mol}^{-1} \mathrm{~cm}^{-1}\right): 202$ (37 730), 228 (21 445), 294 (12 400). IR (diamond) $v_{\max } / \mathrm{cm}^{-1}: 3072(\mathrm{CH}), 1732$ (CO-syd); ${ }^{1} \mathrm{H}$ NMR (300 $\left.\mathrm{MHz}, \mathrm{CDCl}_{3}\right) \delta / \mathrm{ppm}: 7.59(\mathrm{~d}, 1 \mathrm{H}, J=7.8 \mathrm{~Hz}), 7.52(\mathrm{t}, 1 \mathrm{H}, J=7.8 \mathrm{~Hz}), 7.46(\mathrm{t}, 1 \mathrm{H}, J=7.8 \mathrm{~Hz})$, 7.32-7.22 (m, 7H), $7.08(\mathrm{~d}, 2 \mathrm{H}, J=8.5 \mathrm{~Hz}), 6.72(\mathrm{~d}, 2 \mathrm{H}, J=8.5 \mathrm{~Hz}), 6.55(\mathrm{~d}, 1 \mathrm{H}, J=12.1 \mathrm{~Hz}$, H-7/8), 6.03 (d, 1H, $J=12.1 \mathrm{~Hz}, \mathrm{H}-7 / 8) .{ }^{13} \mathrm{C} \mathrm{NMR}\left(150 \mathrm{MHz}, \mathrm{CDCl}_{3}\right) \delta / \mathrm{ppm}: 166.9$ (s, COsyd), 133.9 (d), 133.8 (s), 133.8 (s), 133.6 (s), 133.2 (s), 131.9 (d), 131.3 (d), 129.9 (d), 129.0 (d), 128.8 (d), 128.8 (d), 128.5 (d), 126.8 (d, 2C), 124.7 (s), 122.9 (d), 109.2 (s, C-syd). MS, $m / z$ : $374.89\left(\mathrm{M}^{+}, 100 \%\right), 376.87\left(\mathrm{M}^{+}, 45 \%\right)$.

3-[2-[2-(4-Bromphenyl)ethenyl]phenyl]-4-phenylsydnone (11). Yield 44\%, $185 \mathrm{mg}$. According to ${ }^{1} \mathrm{H}$ NMR spectrum cis/trans $=1 / 0.6$. Dichloromethane was used as eluent.

trans-11. White solid, mp $157-158^{\circ} \mathrm{C}$; UV (EtOH) $\lambda_{\max } / \mathrm{nm}\left(\varepsilon / \mathrm{dm}^{3} \mathrm{~mol}^{-1} \mathrm{~cm}^{-1}\right): 202$ (33 674), 231 (19 055), 315 (31 204). IR (diamond) $v_{\max } / \mathrm{cm}^{-1}: 3062(\mathrm{CH}), 1732$ (CO-syd). ${ }^{1} \mathrm{H}$ NMR (300 $\left.\mathrm{MHz} \mathrm{CDCl}_{3}\right) \delta / \mathrm{ppm}: 7.85(\mathrm{~d}, 1 \mathrm{H}, J=7.7 \mathrm{~Hz}), 7.68(\mathrm{t}, 1 \mathrm{H}, J=7.7 \mathrm{~Hz}), 7.50(\mathrm{t}, 1 \mathrm{H}, J=7.7 \mathrm{~Hz})$, 7.47-7.43 (m, 3H), 7.28-7.14 (m, 7H), $6.95(\mathrm{~d}, 1 \mathrm{H}, J=16.2 \mathrm{~Hz}, \mathrm{H}-7 / 8), 6.65$ (d, 1H, J = 16.2 Hz, H-7/8). ${ }^{13} \mathrm{C}$ NMR (150 MHz, $\mathrm{CDCl}_{3}$ ) $\delta / \mathrm{ppm}: 167.0$ (s, CO-syd), 135.0 (s), 134.0 (s), 133.6 (d), 132.6 (d), 132.6 (s), 132.2 (d), 129.2 (d), 129.0 (d), 128.9 (d), 128.6 (d), 127.4 (d), 127.0 (d), 126.6 (d), 124.7 (s), 123.7 (s), 120.9 (d), 109.5 (s, C-syd). MS, m/z: 418.80 (M+, 100\%), 420.80 $\left(\mathrm{M}^{+}, 100 \%\right)$. Elemental analysis, calcd for $\mathrm{C}_{22} \mathrm{H}_{15} \mathrm{BrN}_{2} \mathrm{O}_{2}(\mathrm{Mr}=419.27): \mathrm{C} 63.02, \mathrm{H} \mathrm{3.61}, \mathrm{N}$ $6.68 \%$; found C 63.00, H 3.73, N 6.87\%. 
cis-11. White solid, mp $165-166^{\circ} \mathrm{C}$; UV (EtOH) $\lambda_{\max } / \mathrm{nm}\left(\varepsilon / \mathrm{dm}^{3} \mathrm{~mol}^{-1} \mathrm{~cm}^{-1}\right): 202$ (41 239), 228 (22 763), 280 (14 402). IR (diamond) $v_{\max } / \mathrm{cm}^{-1}: 3073(\mathrm{CH}), 1732$ (CO-syd). ${ }^{1} \mathrm{H}$ NMR (300 $\left.\mathrm{MHz}, \mathrm{CDCl}_{3}\right) \delta / \mathrm{ppm}: 7.59(\mathrm{~d}, 1 \mathrm{H}, J=7.6 \mathrm{~Hz}), 7.52(\mathrm{t}, 1 \mathrm{H}, J=7.6 \mathrm{~Hz}), 7.46(\mathrm{t}, 1 \mathrm{H}, J=7.6 \mathrm{~Hz})$, $7.32-7.22(\mathrm{~m}, 9 \mathrm{H}), 6.65(\mathrm{~d}, 2 \mathrm{H}, J=8.5 \mathrm{~Hz}), 6.51(\mathrm{~d}, 1 \mathrm{H}, J=12.1 \mathrm{~Hz}), 6.05(\mathrm{~d}, 1 \mathrm{H}, J=12.1 \mathrm{~Hz})$. ${ }^{13} \mathrm{C}$ NMR (75 MHz, $\mathrm{CDCl}_{3}$ ) $\delta / \mathrm{ppm}: 167.1$ (s), 134.3 (s), 134.1 (d), 134.0 (s), 133.4 (s), 132.1 (d), 131.7 (d), 131.6 (d), 131.5 (d), 130.4 (d), 129.2 (d), 129.0 (d), 129.0 (d), 127.0 (d), 124.8 (s), 123.2 (d), 109.4 (s, C-syd). MS, m/z: $418.80\left(\mathrm{M}^{+}, 100 \%\right), 420.80\left(\mathrm{M}^{+}, 100 \%\right)$.

4-Phenyl-3-[2-[2-(4-methoxyphenyl)ethenyl]phenyl]sydnone (12). Yield 71\%, $263 \mathrm{mg}$. According to ${ }^{1} \mathrm{H}$ NMR spectrum cis/trans $=1 / 0.8$. Dichloromethane was used as eluent.

trans-12. Greenish solid, mp 122-123 ${ }^{\circ} \mathrm{C}$; UV (EtOH) $\lambda_{\max } / \mathrm{nm}\left(\varepsilon / \mathrm{dm}^{3} \mathrm{~mol}^{-1} \mathrm{~cm}^{-1}\right): 203$ (34 348), 236 (18 333), 324 (32 496). IR (diamond) $v_{\max } / \mathrm{cm}^{-1}: 3102(\mathrm{CH}), 1765$ (CO-syd). ${ }^{1} \mathrm{H}$ NMR (300 $\left.\mathrm{MHz}, \mathrm{CDCl}_{3}\right) \delta / \mathrm{ppm}$ : $7.85(\mathrm{~d}, 1 \mathrm{H}, J=7.9 \mathrm{~Hz}), 7.65$ (t, $\left.1 \mathrm{H}, J=7.9 \mathrm{~Hz}\right), 7.47-7.39(\mathrm{~m}, 2 \mathrm{H}), 7.30-$ $7.19(\mathrm{~m}, 7 \mathrm{H}), 7.00(\mathrm{~d}, 1 \mathrm{H}, J=16.2 \mathrm{~Hz}, \mathrm{H}-7 / 8), 6.85$ (d, 2H, $J=8.7 \mathrm{~Hz}), 6.53$ (d, 1H, $J=16.2$ $\mathrm{Hz}, \mathrm{H}-7 / 8$ ), 3.82 (s, 3H, $\mathrm{OCH}_{3}$ ). ${ }^{13} \mathrm{C} \mathrm{NMR}\left(75 \mathrm{MHz}, \mathrm{CDCl}_{3}\right.$ ) $\delta / \mathrm{ppm}: 167.0$ (s, CO-syd), 160.5 (s), 134.6 (s), 134.3 (d), 132.5 (d), 132.2 (s), 128.9 (d), 128.9 (d), 128.8 (s), 128.6 (d), 128.4 (d), 127.0 (d), 126.9 (d), 126.5 (d), 124.7 (s), 117.7 (d), 114.5 (d), 109.5 (s, C-syd), 55.6 (q, OCH OC. $_{3}$ MS, $m / z: 370.92\left(\mathrm{M}^{+}, 100 \%\right)$. Elemental analysis, calcd for $\mathrm{C}_{23} \mathrm{H}_{18} \mathrm{~N}_{2} \mathrm{O}_{3}(\mathrm{Mr}=370.4)$ : $\mathrm{C} 74.58$, $\mathrm{H}$ 4.90, N 7.56\%; found C 74.32, H 4.76, N 7.73\%.

cis-12. White solid, mp 124-125 ${ }^{\circ} \mathrm{C}$; UV (EtOH) $\lambda_{\max } / \mathrm{nm}\left(\varepsilon / \mathrm{dm}^{3} \mathrm{~mol}^{-1} \mathrm{~cm}^{-1}\right): 202$ (41 307$), 234$ (22 003), 316 (15 554). IR (diamond) $v_{\max } / \mathrm{cm}^{-1}: 2958,2832(\mathrm{CH}), 1730$ (CO-syd). ${ }^{1} \mathrm{H}$ NMR (600 $\left.\mathrm{MHz} \mathrm{CDCl}_{3}\right) \delta / \mathrm{ppm}: 7.55(\mathrm{~d}, 1 \mathrm{H}, J=7.6 \mathrm{~Hz}), 7.48(\mathrm{t}, 1 \mathrm{H}, J=7.6 \mathrm{~Hz}), 7.45(\mathrm{t}, 1 \mathrm{H}, J=7.6 \mathrm{~Hz})$, $7.39(\mathrm{~d}, 1 \mathrm{H}, J=7.6 \mathrm{~Hz}), 7.32(\mathrm{~d}, 2 \mathrm{H}, J=8.0 \mathrm{~Hz}), 7.25-7.22(\mathrm{~m}, 3 \mathrm{H}), 6.79(\mathrm{~d}, 2 \mathrm{H}, J=8.0 \mathrm{~Hz})$, $6.64(\mathrm{~d}, 2 \mathrm{H}, J=8.0 \mathrm{~Hz}), 6.52(\mathrm{~d}, 1 \mathrm{H}, J=12.0 \mathrm{~Hz}, \mathrm{H}-7 / 8), 5.9(\mathrm{~d}, 1 \mathrm{H}, J=12.0 \mathrm{~Hz}, \mathrm{H}-7 / 8), 3.76$ $\left(\mathrm{s}, 3 \mathrm{H}, \mathrm{OCH}_{3}\right) .{ }^{13} \mathrm{C} \mathrm{NMR}\left(150 \mathrm{MHz}, \mathrm{CDCl}_{3}\right) \delta / \mathrm{ppm}: 167.0$ (s, CO-syd), 159.3 (s), 134.6 (d), 134.6 (s), 133.2 (s), 131.8 (d), 131.4 (d), 130.1 (d), 128.8 (d), 128.6 (d), 128.6 (d), 127.7 (s), 126.7 (d), 126.6 (d), 124.8 (s), 120.3 (d), 113.6 (d), 109.1 (s, C-syd), 55.2 (q, OCH $)$. MS, m/z: $370.92\left(\mathrm{M}^{+}, 100 \%\right)$.

\section{Acknowledgements}

The antiproliferative activity screening was performed at the Laboratory of Experimental Therapy, Division of Molecular Medicine, Rudjer Boskovic Institute, Zagreb. We are thankful to Dr. Marijeta Kralj and Dr. Lidija Šuman for experimental and financial support (the grant from the Ministry of Science, Education and Sports of the Republic of Croatia 098- 0982464-2514). This work was supported by grants from the Ministry of Science, Education and Sports of the Republic of Croatia (grant no. 125-0982933-2926, 098-0982929-2917). 


\section{References}

1. For general overviews of the chemistry of sydnones see: (a) Stewart, F. H. C. Chem. Rev. 1964, 64, 129. (b) Newton, C. G.; Ramsden, C. A. Tetrahedron 1982, 38, 2965. (c) Clapp, L. B. In Comprehensive Heterocyclic Chemistry; Katritzky, A. R., Ed.; Pergamon: Oxford, 1984; 4B; Vol. 6, p 365. (d) Gribble, G. W. In Synthetic Applications of 1,3-Dipolar Cycloaddition Chemistry toward Heterocycles and Natural Products; Padwa, A., Pearson, W. H., Eds.; Wiley: New Jersey, NJ, 2003; p 681. (e) Browne, D. L.; Harrity, J. P. A. Tetrahedron 2010, 66, 553.

2. Earl, J. C.; Mackney, A. W. J. Chem. Soc. 1935, 899.

3. Bos, M.; Fleischhacker, W. Pharmazie in unserer Zeit 1984, 13, 51.

4. Moustafa, M. A.; Gineinah, M. M.; Nasr, M. N.; Bayoumi, W. A. H. Arch. Pharmacol. 2004, 337, 427.

5. (a) Dunkley, C. S.; Thoman, C. J. Bioorg. Med. Chem. Lett. 2003, 13, 2899. (b) Grynberg, N.; Gomes, R.; Shinzato, T.; Echevarria, A.; Miller, J. Anticancer Res. 1992, 12, 1025.

6. (a) Wagner, H.; Hill, J. B. J. Med. Chem. 1974, 17, 1337. (b) Hill, J. B.; Ray, R. E.; Wagner, H.; Aspinall, R. L. J. Med. Chem. 1975, 18, 50.

7. Becher, K. B. Synthesis 1983, 341.

8. Dorrie, J.; Gerauer, H.; Wachter, Y.; Zunino, S. J. Cancer Res. 2001, 61, 4731.

9. Lin, C. M.; Ho, H. H.; Pettit G. R., Hamel, E. Biochemistry 1989, $28,6984$.

10. (a) Butković, K.; Vuk, D.; Marinić, Ž.; Penić, J.; Šindler-Kulyk, M. Tetrahedron 2010, 66, 9356; (b) Butković, K; Marinić, Ž.; Šindler-Kulyk, M. Magn. Reson. Chem. 2004, 42, 1053.

11. Preston, P. N.; Turnbull, K. J. Chem. Soc. Perkin Trans. 1 1977, 1229.

12. Lowe, J. D.; Turnbull, K. J. Heterocyclic Chem. 1986, 25, 125.

13. Xiao, C. F.; Tao, L. Y.; Sun, H Y.; Wei W.; Chen Y.; Fu L W.; Zou, Y. Chinese Chem. Lett. 2010, 21, 1295.

14. Murias, M.; Jager W.; Handler, N.; Erker, T.; Horvath Z.; Szekeres, T.; Nohl, H.; Gille, L. Biochem. Pharmacol. 2005, 69, 903.

15. Vorlaender, D.; Von Schilling, R. Chem. Ber. 1900, 33, 553. 